\title{
Five Crucial Challenges in Digital Health
}

\author{
Nicholas Cummins ${ }^{1,2}$ and Björn W. Schuller ${ }^{1,3 *}$ \\ ${ }^{1}$ Chair of Embedded Intelligence for Health Care and Wellbeing, University of Augsburg, Augsburg, Germany, ${ }^{2}$ The \\ Department of Biostatistics and Health Informatics, Institute of Psychiatry, Psychology and Neuroscience, King's College \\ London, London, United Kingdom, ${ }^{3}$ Department of Computing, Imperial College London, London, United Kingdom
}

Keywords: positioning, overview, survey, grand challenge, digital health

\section{INTRODUCTION}

The concept of digital health is not a new or revolutionary one. For example, technologies, such as medical images and telemedicine date back over 100 years $(1,2)$, while prototype wearable devices have been used to tackle obesity since the 1940s (3). Digital health, however, has had continually transforming effects in an industry that is notoriously resistant to change (4). Moreover, since the mid-1990s when the US National Academy of Medicine began recommending the complete digitization of health data (5), the transforming effects of digital technologies in healthcare has never been more evident. With the advent of wearables and other Internet of Things (IoT) devices, health care is moving further toward personalized and preventative paradigms utilizing ubiquitous technologies which support real-time self-care or monitoring (6).

The ongoing coronavirus disease 2019 (COVID-19) pandemic increases the need for ongoing digital health advancements. For example, traditional face-to-face medical consults increase the risk of infection, emphasizing the need for virtual consultation technologies (7). Similarly, tools are needed to help understand and support the effects of the pandemic on our physical and, in particular, mental health (8). Even without this amplified need, it is clear that digital technologies will continue to transform the healthcare sector again and again. As with Wilhelm Conrad Röntgen-the physicist who discovered X-rays (1) - and many others before, contributions are expected from individuals with technical backgrounds rather than just pure medical. Building on from Kostkova (9), this article highlights a selection of crucial current challenges to overcome to ensure that digital health systems to meet the guiding principle of being for all anywhere

OPEN ACCESS

Edited and reviewed by: Constantinos S. Pattichis, University of Cyprus, Cyprus

${ }^{*}$ Correspondence: Björn W. Schuller schuller@ieee.org

Received: 30 September 2020 Accepted: 04 November 2020 Published: 08 December 2020

Citation:

Cummins N and Schuller BW (2020) Five Crucial Challenges in Digital Health

Front. Digit. Health 2:536203. doi: 10.3389/fdgth.2020.536203 and anytime.

\section{SOCIETAL FACTORS}

While technology is at the heart of any digital health system, the related transformations cannot be viewed purely through a technological lens (10). Digital technologies need to deliver affordable, easy-to-use healthcare solutions to a growing and aging population in which new technologies are often slow to be adopted and accepted by the general populace (11). Factors that influence this lack of acceptance include regulatory factors, such as uncertainties surrounding digital health policies and legislation as well as a perceived lack of accountability within the commercial sector. The commercial sector itself has challenges related to the complexity of the multinational nature of the digital health market and the need to operate within the constraints of a multitude of different health systems. Low levels of digital and health literacy-and thus digital health literacy-in the general population, especially in the elderly, is also a major contributing factor.

Substantial efforts, therefore, needed to be made to normalize the use of digital health at a societal level. These efforts should include upskilling both health professional and the general public through broad-reaching educational initiatives (11). Processes are also needed to assess 
the suitability of digital health solutions from the perspective of all stakeholders, especially those representing the patient and clinician end-users groups (12). To ensure wider adaption, the next generation of digital technologies should support and engage users in such a way that fosters equality and inclusivity, resulting in improved healthcare solutions for all $(13,14)$.

COVID-19 has highlighted the need to adapt and update clinical care delivery systems (15). Concerns have been raised that efforts to move away from traditional face-to-face medicine and toward remote, digital, solutions have highlighted, such as the already existing socioeconomic gaps between groups of people who can easily access and use such services and those who cannot $(16,17)$. Social media, while playing a vital role in supporting communication between social and family groups during lockdowns, has also made it considerably easier to spread medical misinformation across societies $(18,19)$. Societal and governmental efforts are urgently needed to help counter this negative phenomenon (19). Contact tracing using remote apps has enabled mass data collection to aid public health and research efforts (16). However, this raises concerns relating to data ownership and other ethical concerns $(16,20)$.

\section{ETHICAL CHALLENGES}

The increasing digitization of healthcare and the growth of mobile and IoT devices as data collection tools raises many ethical issues. One commonly recurring theme relates to the exact nature of the role of consumer tech companies, such as Amazon, Apple, Google, Facebook, or Samsung, who have all entered the digital health domain (21). In particular, such companies offer solutions for collecting, storing and analyzing health data which raises issues relating to privacy, data protection and informed consent (21-23). The nature of health data is also changing; we are now collecting more private user-generated data, particularly data harvested from social media and through wearable technologies, than ever before.

As well as the issues mentioned above of privacy, protection, and consent, ethical concerns relating to data ownership are frequently discussed in the literature [e.g., $(23,24)]$. The growth of apps and technologies developed for a consumer market blurs the lines between what is medical and non-medical devices and raises ethical challenges relating to how to regularize such technologies $(22,25)$. This issue is exacerbated by the speed of advancements and increasing globalization of healthcare solutions $(25,26)$.

\section{INCREASED CONNECTED HEALTH SOLUTIONS}

A core aim of digital health is to help facilitate the circulation of data between patients, devices, and clinicians (27). Increasing this connectivity enables smarter and more timely sharing of information between clinicians and patients and has strong links to the predictive, preemptive, and personalized principles of digital health (28). Connected health solutions are also a key element in response to the COVID-19 pandemic (29). However, increased connected health solutions come with increased safety and security concerns.

As medicine moves beyond conventional clinical-based patient care to the personalized and preventative models offered by digital health applications, our concepts of patient safety will also have to change $(30,31)$. The current speed of technological advances also brings with it safety concerns. There is often a lack of quality and evidence-based research highlighting the associated health benefits of the newest technologies. There are also many challenges inherent in demonstrating that newer approaches and technologies, are indeed, effective (32).

The use of IoT devices in connected health means greater support for anywhere anytime solutions as well as real-time selfcare or monitoring (33). However, transferring data from the point of collection, such as IoT devices, to remote serves, brings security and privacy concerns that need to be addressed (34). While the practices of informed consent and privacy by design are well-established in digital health, there are still concerns surrounding patients' understanding of how their data is being processed and by whom (35). An emerging trend, not just in digital health, but in health research in general, is increased patient engagement, treating the patient as a stakeholder in research, not just a data source (36). The perspective of patients is vital for gaining a real understanding of what security and privacy mean in the context of connected health (37).

At the time of writing, there is no universally established treatment or vaccine for COVID-19, the IoT can play a vital role as an information source and monitoring tool during the pandemic $(38,39)$ or potentially oncoming ones. Moreover, such devices can help in efforts to nowcast events, such as second waves via the harnessing of large amounts of physiological and behavioral data gathered from the general population (40). However, the collection of such data is not enough; complimentary artificial intelligence solutions are needed to exploit the saliency of such data (41).

\section{ROLE OF ARTIFICIAL INTELLIGENCE}

Artificial Intelligence can utilize data generated in digital health systems to help with aspects in medicine, such as improved diagnosis, selecting treatments, and predicting clinical outcomes. The presence of AI solutions in digital health intensifies challenges surrounding safety, explainability, and fairness (42). In regard to safety, AI systems are held to higher perceived safety standards than humans (43); i.e., it is less acceptable for AI to make errors. Moreover, the risk to human life of AI-based systems is, currently, not well-studied, and there is a lack of standards for the verification and validation of such systems. There are also generalization issues associated with AI models, reproducing promising results, made on "limited" training sets, on real-world data. A recent systematic review of deep learning solutions in medical images found that only a minimal number of studies in this field were of sufficient quality for clinical implementation (44). 
With the recent introduction of the General Data Protection Regulation (GDPR) in the European Union, clinicians and patients have a right to understand how a particular AI decision was reached (45). The benefits of improving trust and transparency in AI systems will not only benefit clinicians and patients; increased knowledge and understanding into internal operations and decisions should also improve the overall accuracy and generalizability of the enhanced system (46). Therefore, for deployment in clinical settings, the next generation of AI technologies needs to be transparent, understandable, explainable, and fair.

Despite AI already having achieved remarkable results in a range of health-based detection tasks, increasing explainability is a highly non-trivial task. This difficulty arises as many of these results have been achieved using "black-box" techniques. That is, data is fed in, which in turn generates a predictive output, but the system does not provide any information or inference concerning how it arrived at the predicted value. This issue is particularly pronounced with the increasing presence of deep learning systems in digital health (47). Deep learning models have internal connections measuring in the millions (48).

\section{THE POTENTIAL OF GENOMICS}

Technological advances, increasing demand, and a reduction in costs mean that the amount of people undertaking genetic profiles is increasing. Despite this increase in supply and demand, outside of a handful of notable cases, such as rare disease diagnosis and cancer screening, genetic information is not integrated into routine medical care $(49,50)$. Genomics information has the potential to make considerable gains in data-driven, personalized care (51). For this potential to be met, there needs to be further developments in genetic risk scores relevant for broader clinical, and greater understandings into the interpretation of genetic variants (49).

These two factors are, of course, interlinked. Genomics, on one level, essentially provides information regarding "what might happen" to an individual concerning their health (52). A key factor in improving our understanding of this information is the interpretation of genetic variants identified during testing. There are many millions of these variants, and no standard definition for all of them $(50,53)$. Facilitating data and computational resource sharing are commonly identified means of closing this knowledge gap (50). Data sharing comes with increased ethical concerns, which have already been discussed in this article.

Genomics advancements are interlinked connected health challenges. For truly personalized medical care, genomics information should be combined with environmental, behavioral, and medical history information. Moreover, this combination of information from multiple heterogeneous sources needs to be performed in such a way that supports, not confounds clinicians and patients. These heterogeneous systems are highly dynamic and will most likely require advanced artificial intelligence paradigms to fully combine and analyse $(49,54)$.

Finally, genomics will play a vital role in efforts combating COVID-19, and any future similar pandemics. Increased genomic sequencing capabilities, accelerated through the use of $\mathrm{AI}$, can help in the tracking of pathogens and viruses, and in the identification of their genomic signatures (55). Improved capabilities can also help identify genomic factors that increase an individual's susceptibility or resistance (56).

\section{CONCLUSION}

The current coronavirus disease 2019 (COVID-19) pandemic is undoubtedly challenging conventional medical services to their core. Digital health solutions are going to play a vital role in fighting the pandemic and potential future ones by enabling fundamental shifts in medical care both during the pandemic and in the aftermath. Such advancements are not going to come without challenges, both relating directly to the pandemic and more broadly to the development and implementation of new digital health solutions. Five years ago, Multi-disciplinary Digital Health, Big Data and Public Health, MedTech, Self-Management, and Personalized Care, mHealth and Global Health Interventions, Evidence and Knowledge: Semantics, Social Media, and Persuasion, Serious Health Games and Games-Based Learning and Training, and Personal and Population Data-To Share or Not to Share? were identified as some of the major challenges in digital health (9). These challenges are still very much at the forefront of digital health research. Rather than rehashing these challenges, this article has highlighted new concerns, with a focus on the role of digital health concerning COVID-19 and contagious virus diseases. By highlighting these challenges, Frontiers in Digital Health hopes to continue the trend of publishing worldclass multi-disciplinary research addressing these and many other challenges in this exciting and constantly evolving field of research.

\section{AUTHOR CONTRIBUTIONS}

All authors listed have made a substantial, direct and intellectual contribution to the work, and approved it for publication.

\section{FUNDING}

The author acknowledges support from the European Union's Horizon 2020 research and innovation programme under grant agreement No. 826506 (sustAGE), and from the Innovative Medicines Initiative 2 Joint Undertaking under grant agreement No. 115902. This joint undertaking receives support from the European Union's Horizon 2020 research and innovation programme and EFPIA. 


\section{REFERENCES}

1. Bradley WG. History of medical imaging. Proc Am Philos Soc. (2008) 152:349-61. Available online at: http://www.umich.edu/ ners580/nersbioe_481/lectures/pdfs/2008-09-procAmerPhilSoc_Bradley-MedicalImaging History.pdf

2. Darkins A, Darkins AW, Darkins WA, Cary MA, Cary M. Telemedicine and Telehealth: Principles, Policies, Performances and Pitfalls. New York, NY: Springer Publishing Company (2000).

3. Arigo D, Jake-Schoffman DE, Wolin K, Beckjord E, Hekler EB, Pagoto SL. The history and future of digital health in the field of behavioral medicine. J Behav Med. (2019) 42:67-83. doi: 10.1007/s10865-018-9966-z

4. Metcalf D, Milliard ST, Gomez M, Schwartz M. Wearables and the internet of things for health: Wearable, interconnected devices promise more efficient and comprehensive health care. IEEE Pulse. (2016) 7:35-9. doi: 10.1109/MPUL.2016.2592260

5. Evans R. Electronic health records: then, now, and in the future. Yearb Med Inform. (2016) 25:S48-61. doi: 10.15265/IYS-2016-s006

6. Qi J, Yang P, Min G, Amft O, Dong F, Xu L. Advanced internet of things for personalised healthcare systems: a survey. Perv Mobile Comput. (2017) 41:132-49. doi: 10.1016/j.pmcj.2017.06.018

7. Webster P. Virtual health care in the era of COVID-19. Lancet. (2020) 395:1180-1. doi: 10.1016/S0140-6736(20)30818-7

8. Holmes EA, O'Connor RC, Perry VH, Tracey I, Wessely S, Arseneault L, et al. Multidisciplinary research priorities for the COVID-19 pandemic: a call for action for mental health science. Lancet Psychiatry. (2020) 7:547-560. doi: 10.1016/S2215-0366(20)30168-1

9. Kostkova P. Grand challenges in digital health. Front Public Health. (2015) 3:134. doi: 10.3389/fpubh.2015.00134

10. Aickelin U, Chapman WW, Hart GK. Health Informatics-ambitions and purpose. Front Digit Health. (2019) 1:a2. doi: 10.3389/fdgth.2019.00002

11. Lennon MR, Bouamrane MM, Devlin AM, O'Connor S, O'Donnell C, Chetty U, et al. Readiness for delivering digital health at scale: lessons from a longitudinal qualitative evaluation of a national digital health innovation program in the United Kingdom. J Med Internet Res. (2017) 19:e4. doi: 10.2196/jmir.6900

12. Flott K, Callahan R, Darzi A, Mayer E. A patient-centered framework for evaluating digital maturity of health services: a systematic review. J Med Internet Res. (2016) 18:e75. doi: 10.2196/jmir.5047

13. Robbins D, Dunn P. Digital health literacy in a person-centric world. Int J Cardiol. (2019) 290:154-5. doi: 10.1016/j.ijcard.2019.05.033

14. Alcaraz KI, Sly J, Ashing K, Fleisher L, Gil-Rivas V, Ford S, et al. The ConNECT framework: a model for advancing behavioral medicine science and practice to foster health equity. J Behav Med. (2017) 40:23-38. doi: 10.1007/s10865-016-9780-4

15. Keesara S, Jonas A, Schulman K. Covid-19 and health care's digital revolution. N Engl J Med. (2020) 382:e82. doi: 10.1056/NEJMp2005835

16. Gasser U, Ienca M, Scheibner J, Sleigh J, Vayena E. Digital tools against COVID-19: taxonomy, ethical challenges, and navigation aid. Lancet Digit Health. (2020) 2:e425-34. doi: 10.1016/S2589-7500(20)30137-0

17. Ramsetty A, Adams C. Impact of the digital divide in the age of COVID-19. J Am Med Inform Assoc. (2020) 27:1147-8. doi: 10.1093/jamia/ocaa078

18. Laato S, Islam AKMN, Islam MN, Whelan E. What drives unverified information sharing and cyberchondria during the COVID-19 pandemic? Eur J Inform Syst. (2020) 29:288-305. doi: 10.1080/0960085X.2020.1770632

19. Pennycook G, McPhetres J, Zhang Y, Lu JG, Rand DG. Fighting COVID-19 misinformation on social media: experimental evidence for a scalable accuracy-nudge intervention. Psychol Sci. (2020) 31:770-80. doi: 10.1177/0956797620939054

20. Fahey RA, Hino A. COVID-19, digital privacy, and the social limits on datafocused public health responses. Int J Inform Manage. (2020) 55:102181. doi: 10.1016/j.ijinfomgt.2020.102181

21. Sharon T. The Googlization of health research: from disruptive innovation to disruptive ethics. Pers Med. (2016) 13:563-74. doi: 10.2217/pme-2016-0057

22. Schmietow B, Marckmann G. Mobile health ethics and the expanding role of autonomy. Med Health Care Philos. (2019) 22:623-30. doi: 10.1007/s11019-019-09900-y
23. Mirchev M. Patient information ownership in the age of digital health and big data. Eur J Public Health. (2019) 29:ckz186.078. doi: 10.1093/eurpub/ckz186.078

24. Kostkova P, Brewer H, de Lusignan S, Fottrell E, Goldacre B, Hart G, et al. Who owns the data? Open data for healthcare. Front Public Health. (2016) 4:7. doi: 10.3389/fpubh.2016.00007

25. Cortez N. 18. In: The Evolving Law and Ethics of Digital Health. Cham: Springer International Publishing (2018). p. 249-69. doi: 10.1007/978-3-319-61446-5_18

26. Ruotsalainen PS. Chapter 5-privacy, trust and security in two-sided markets. In: Vimarlund V, editor. E-Health Two-Sided Markets. Academic Press (2017). p. 65-89. doi: 10.1016/B978-0-12-805250-1.00005-8

27. Vayena E, Haeusermann T, Adjekum A, Blasimme A. Digital health: meeting the ethical and policy challenges. Swiss Med Weekly. (2018) 148:w14571. doi: 10.4414/smw.2018.14571

28. Pattichis CS, Panayides AS. Connected health. Front Digit Health. (2019) 1:1. doi: 10.3389/fdgth.2019.00001

29. Radanliev P, De Roure D, Walton R, Van Kleek M, Montalvo RM, Santos O, et al. COVID-19 what have we learned? The rise of social machines and connected devices in pandemic management following the concepts of predictive, preventive and personalized medicine. EPMA J. (2020) 11:311-32. doi: 10.2139/ssrn.3692585

30. Agboola SO, Bates DW, Kvedar JC. Digital health and patient safety. JAMA. (2016) 315:1697-8. doi: 10.1001/jama.2016.2402

31. Mathews SC, McShea MJ, Hanley CL, Ravitz A, Labrique AB, Cohen AB. Digital health: a path to validation. NPJ Digit Med. (2019) 2:38. doi: 10.1038/s41746-019-0111-3

32. Patrick K, Hekler EB, Estrin D, Mohr DC, Riper H, Crane D, et al. The pace of technologic change: implications for digital health behavior intervention research. Am J Prev Med. (2016) 51:816-24. doi: 10.1016/j.amepre.2016.05.001

33. Baker SB, Xiang W, Atkinson I. Internet of things for smart healthcare: technologies, challenges, and opportunities. IEEE Access. (2017) 5:26521-44. doi: 10.1109/ACCESS.2017.2775180

34. Yang Y, Wu L, Yin G, Li L, Zhao H. A survey on security and privacy issues in internet-of-things. IEEE Internet Things J. (2017) 4:1250-8. doi: 10.1109/JIOT.2017.2694844

35. O'Connor Y, Rowan W, Lynch L, Heavin C. Privacy by design: informed consent and internet of things for smart health. Proc Comput Sci. (2017) 113:653-8. doi: 10.1016/j.procs.2017.08.329

36. de Wit M, Cooper C, Reginster JY. Practical guidance for patient-centred health research. Lancet. (2019) 393:1095-6. doi: 10.1016/S0140-6736(19)30034-0

37. Taylor MJ, Wilson J. Reasonable expectations of privacy and disclosure of health data. Med Law Rev. (2019) 27:432-60. doi: 10.1093/medlaw/fwz009

38. Singh RP, Javaid M, Haleem A, Suman R. Internet of things (IoT) applications to fight against COVID-19 pandemic. Diabetes Metab Syndr Clin Res Rev. (2020) 14:521-4. doi: 10.1016/j.dsx.2020.04.041

39. Sun S, Folarin AA, Ranjan Y, Rashid Z, Conde P, Stewart C, et al. Using smartphones and wearable devices to monitor behavioural changes during COVID-19. J Med Internet Res. (2020) 22:e19992. doi: 10.2196/ 19992

40. Radin JM, Wineinger NE, Topol EJ, Steinhubl SR. Harnessing wearable device data to improve state-level real-time surveillance of influenza-like illness in the USA: a population-based study. Lancet Digit Health. (2020) 2:e85-93. doi: 10.1016/S2589-7500(19)30222-5

41. Ting DSW, Carin L, Dzau V, Wong TY. Digital technology and COVID-19. Nat Med. (2020) 26:459-61. doi: 10.1038/s41591-020-0824-5

42. Panch T, Mattie H, Atun R. Artificial intelligence and algorithmic bias: implications for health systems. J Glob Health. (2019) 9:010318. doi: $10.7189 /$ jogh.09.020318

43. Winfield A. Ethical standards in robotics and AI. Nat Electron. (2019) 2:46-8. doi: 10.1038/s41928-019-0213-6

44. Liu X, Faes L, Kale AU, Wagner SK, Fu DJ, Bruynseels A, et al. A comparison of deep learning performance against health-care professionals in detecting diseases from medical imaging: a systematic review and metaanalysis. Lancet Digit Health. (2019) 1:e271-97. doi: 10.1016/S2589-7500(19) 30123-2 
45. Voigt P, Von dem Bussche A. The EU General Data Protection Regulation (GDPR): A Practical Guide. Cham: Springer (2017).

46. Adadi A, Berrada M. Peeking inside the black-box: a survey on explainable artificial intelligence (XAI). IEEE Access. (2018) 6:52138-60. doi: 10.1109/ACCESS.2018.2870052

47. Cummins N, Schuller BW, Baird A. Speech analysis for health: current state-of-the-art and the increasing impact of deep learning. Methods. (2018) 151:41-54. doi: 10.1016/j.ymeth.2018. 07.007

48. Han S, Pool J, Tran J, Dally WJ. Learning both weights and connections for efficient neural networks. In: Cortes C, Lawrence ND, Lee DD, Sugiyama M, Garnett R, editors. Proceedings of the 28th International Conference on Neural Information Processing Systems, Vol. 1. Montreal, QC: Curran Associates, Inc. (2015). p. 1135-43.

49. Muse ED, Torkamani A, Topol EJ. When genomics goes digital. Lancet. (2018) 391:2405. doi: 10.1016/S0140-6736(18)31272-8

50. Rehm HL. Evolving health care through personal genomics. Nat Rev Genet. (2017) 18:259-67. doi: 10.1038/nrg.2016.162

51. Tarkkala H, Helén I, Snell K. From health to wealth: the future of personalized medicine in the making. Futures. (2019) 109:142-52. doi: 10.1016/j.futures.2018.06.004

52. Moore J. From personalised nutrition to precision medicine: the rise of consumer genomics and digital health. Proc Nutr Soc. (2020) 79:300-10. doi: $10.1017 /$ S0029665120006977
53. The 1000 Genomes Project Consortium. A global reference for human genetic variation. Nature. (2015) 526:68-74. doi: 10.1038/nature15393

54. Xu J, Yang P, Xue S, Sharma B, Sanchez-Martin M, Wang F, et al. Translating cancer genomics into precision medicine with artificial intelligence: applications, challenges and future perspectives. Hum Genet. (2019) 138:109-24. doi: 10.1007/s00439-019-01970-5

55. Randhawa GS, Soltysiak MPM, El Roz H, de Souza CPE, Hill KA, Kari L. Machine learning using intrinsic genomic signatures for rapid classification of novel pathogens: COVID-19 case study. PLoS ONE. (2020) 15:e0232391. doi: 10.1371/journal.pone.0232391

56. Murray MF, Kenny EE, Ritchie MD, Rader DJ, Bale AE, Giovanni MA, et al. COVID-19 outcomes and the human genome. Genet Med. (2020) 22:1175-7. doi: $10.1038 / \mathrm{s} 41436-020-0832-3$

Conflict of Interest: The authors declare that the research was conducted in the absence of any commercial or financial relationships that could be construed as a potential conflict of interest.

Copyright $\odot 2020$ Cummins and Schuller. This is an open-access article distributed under the terms of the Creative Commons Attribution License (CC BY). The use, distribution or reproduction in other forums is permitted, provided the original author(s) and the copyright owner(s) are credited and that the original publication in this journal is cited, in accordance with accepted academic practice. No use, distribution or reproduction is permitted which does not comply with these terms. 